\section{Successful treatment with rituximab in a patient with lupus cerebritis and posterior reversible encephalopathy syndrome: a case report}

\author{
Saemi Choi, MD; Young Seo Kim, MD, PhD \\ Department of Neurology, Hanyang University Hospital, Hanyang University College of Medicine, \\ Seoul, Korea
}

\section{Journal of Neurocritical \\ CASE REPORT \\ Received: October 18, 2021 \\ Revised: October 27, 2021 \\ Accepted: October 28, 2021 \\ Corresponding Author: \\ Young Seo Kim, MD, PhD \\ Department of Neurology, Hanyang \\ University College of Medicine, 222-1 \\ Wangsimni-ro, Seongdong-gu, Seoul \\ 04763, Korea \\ Tel.: +82-2-2290-8378 \\ Fax: +82-2-2299-2391 \\ E-mail: aescula@hanmail.net}

\begin{abstract}
Background: Neuropsychiatric systemic lupus erythematosus (NPSLE) has a diverse and broad spectrum of severity and prognosis, with some devastating manifestations. However, its diagnosis and treatment remain unclear and controversial.

Case Report: A 19-year-old woman with SLE presented with fever, headache, quadriparesis, and tremor. Brain magnetic resonance imaging (MRI) showed sulcal enhancement in the cortical sulcus, and intravenous methylprednisolone $(500 \mathrm{mg} /$ day) and immunoglobulin ( $2 \mathrm{~g} / \mathrm{kg}$ for 5 days) were started under the suspicion of aseptic lupus meningitis. However, the patient's neurologic symptoms worsened; brain MRI showed a newly developed brain parenchymal lesion, suggesting lupus cerebritis and posterior reversible encephalopathy syndrome. Two cycles of rituximab (850 mg/day, 1-week interval) were administered for the treatment of refractory NPSLE. Her neurologic symptoms gradually improved after the second cycle, and she was discharged with minimal neurologic symptoms. Conclusion: Rituximab may be a therapeutic option for refractory lupus cerebritis. Further research is needed to accurately determine its efficacy.
\end{abstract}

Keywords: Neuropsychiatric systemic lupus erythematosus; Posterior reversible encephalopathy syndrome; Rituximab

\section{INTRODUCTION}

Systemic lupus erythematosus (SLE) is an autoimmune disease that can affect any organ in the body. Neuropsychiatric involvement in SLE is common, and its estimated prevalence ranges from $37 \%$ to $95 \%$ [1]. The diagnosis of neuropsychiatric SLE (NPSLE) was proposed by American College of Rheumatology in 1999 for 19 categories of NPSLE that included central nervous system manifestations, such as aseptic meningitis, cerebrovascular dis- ease, demyelinating disease, headache, movement disorder, myelopathy, seizure disorder, cognitive dysfunction, mood disorder, and psychosis, as well as peripheral nervous system manifestations, such as acute inflammatory demyelinating polyradiculoneuropathy, autonomic disorder, mononeuritis, myasthenia gravis, cranial neuropathy, plexopathy, and polyneuropathy [2]. More recently, neuropsychiatric syndromes such as cerebral venous thrombosis, posterior reversible encephalopathy syndrome (PRES), optic neuritis, progressive multifocal leukoencephalopa-

(C) 2021 The Korean Neurocritical Care Society

This is an Open Access article distributed under the terms of the Creative Commons Attribution Non-Commercial License (http://creativecommons.org/licenses/by-nc/4.0/) which permits unrestricted noncommercial use, distribution, and reproduction in any medium, provided the original work is properly cited. 
thy, and idiopathic intracranial hypertension have been added to the classification of NPSLE $[3,4]$. As stated above, the clinical manifestations of NPSLE are diverse and heterogeneous, and NPSLE remains a diagnostic and therapeutic challenge [3]. Furthermore, the occurrence of similar phenomena because of steroid treatment or concomitant diseases such as hypertension and hyperlipidemia makes the diagnosis of NPSLE more difficult [4].

Lupus cerebritis is a poorly defined disease entity, described by some researchers as a former term for NPSLE [3]. However, others have referred the evidence of cerebral parenchymal damage with cerebrospinal fluid (CSF) inflammation in the absence of stroke and vasculitis as lupus cerebritis [5]. Herein, we report a severe case of lupus cerebritis that was treated successfully with rituximab.

\section{CASE REPORT}

A 19-year-old woman presented to the emergency department with fever, headache, nausea, quadriparesis, and tremor. She had been diagnosed with SLE 19 months previously with the findings of discoid rash, non-erosive arthritis, leukopenia, low complement level, positive anti-dsDNA, positive anti-Smith antibody, and negative antiphospholipid antibodies, and she was treated with oral prednisolone at a dose of $15 \mathrm{mg} /$ day and hydroxychloroquine at a dose of $200 \mathrm{mg}$ /day. She had been experiencing inattention, anxiety, insomnia, compulsive behavior (frequent hand washing), tremor, and apraxia for 10 days before admission. Neurologic examination revealed decreased muscle strength in all extremities (Medical Research Council [MRC] grade 4) with normoactive deep tendon reflexes. She also had bulbar symptoms such as dysarthria, dysphagia, and dysphonia accompanied by jaw weakness. Initial brain magnetic resonance imaging (MRI) showed normal fluid-attenuated inversion recovery (FLAIR) imaging (Fig. 1A) with sulcal enhancement in cortical sulcus and cerebellar folia on FLAIR enhancement study (Fig. 1B). Laboratory findings showed leukopenia $\left(3,000 / \mathrm{mm}^{3}\right)$, normal platelet count $\left(211,000 / \mathrm{mm}^{3}\right)$, increased erythrocyte sedimentation rate $(56 \mathrm{~mm} / \mathrm{hr})$, normal C-reactive protein level $(<0.3 \mathrm{mg}$ ) $\mathrm{dL})$, negativity for antiphospholipid antibodies, anti-dsDNA antibody negativity, and normal complement level (C3, $94 \mathrm{mg}$ / $\mathrm{dL} ; \mathrm{C} 4,19.4 \mathrm{mg} / \mathrm{dL}$ ), indicating rather low disease activity. CSF analysis revealed normal opening pressure $\left(120 \mathrm{mmH}_{2} \mathrm{O}\right)$, increased white blood cell count $\left(9 / \mathrm{mm}^{3}\right)$, elevated protein level $(93 \mathrm{mg} / \mathrm{dL})$, and lower glucose level $(69 \mathrm{mg} / \mathrm{dL})$ compared to serum glucose level $(126 \mathrm{mg} / \mathrm{dL})$. Under the suspicion of aseptic meningitis due to SLE, methylprednisolone was started at a dose of $500 \mathrm{mg}$ /day; however, 2 days after starting methylpred-
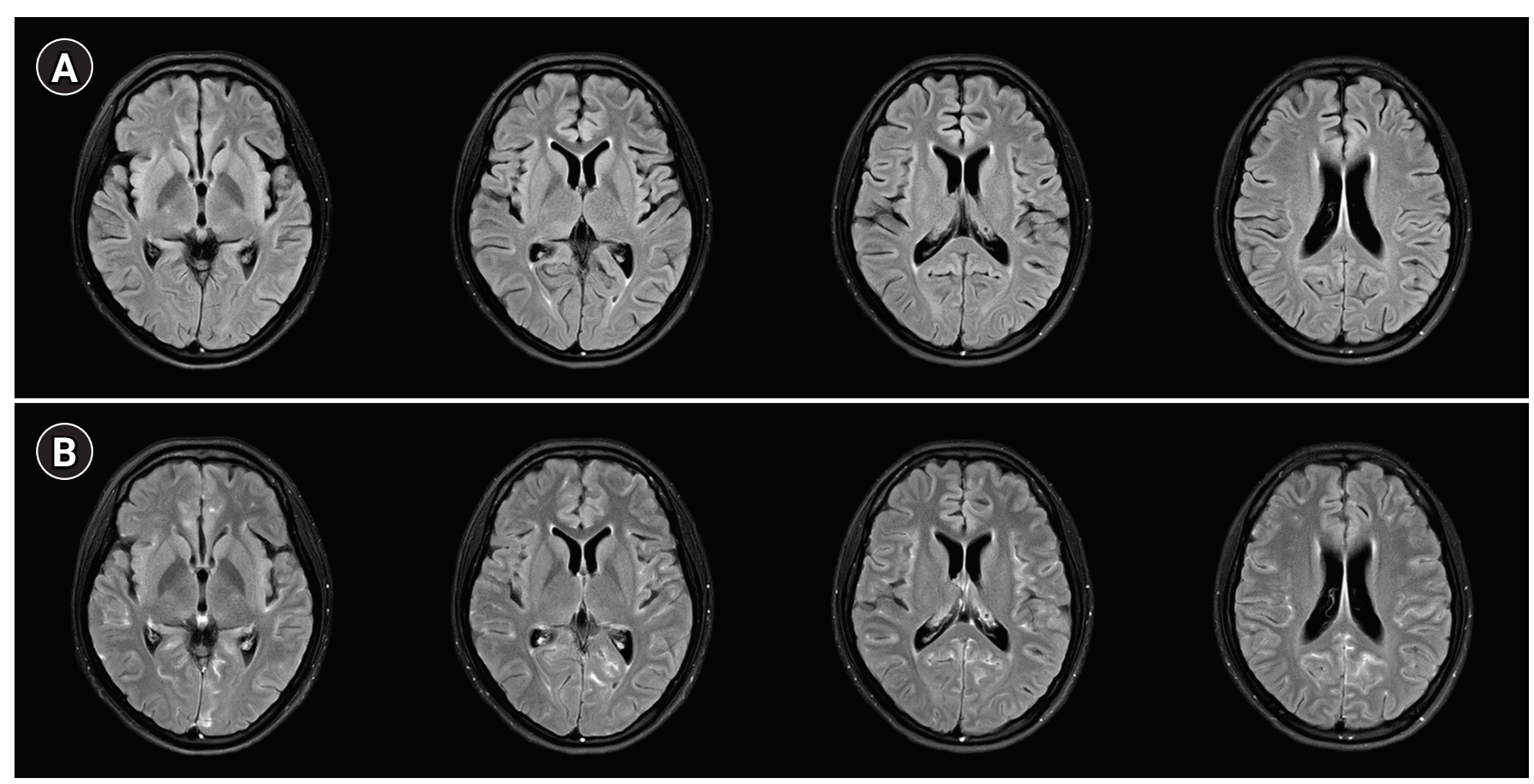

Fig. 1. Initial brain magnetic resonance imaging. (A) Fluid-attenuated inversion recovery (FLAIR) image revealed no abnormal signal intensities. (B) FLAIR-enhanced image revealed multifocal sulcal enhancement in the cortical sulcus, suggesting lupus meningitis. 
nisolone, her tremor and the weakness of the extremities worsened, and she was treated with intravenous immunoglobulin (2 $\mathrm{g} / \mathrm{kg} /$ day) for 5 days along with steroid. On the fifth day of immunoglobulin administration, her vital signs became unstable; blood pressure increased to $153 / 129 \mathrm{mmHg}$, and pulse rate increased to 160 beats per minute without improvement of the neurologic symptoms. Additional brain MRI revealed high signal intensities in the occipital region on T2 and FLAIR imaging, suggesting PRES, as well as focal high signal in the right basal ganglia. Moreover, no basal ganglia lesion was observed on diffusion-weighted imaging and normal angiography. These findings suggested the diagnosis of lupus cerebritis and PRES induced by the disease itself or immunoglobulin (Fig. 2). She re- ceived symptomatic treatment for hypertension and tachycardia. Simultaneously, she was treated with two cycles of rituximab $(850 \mathrm{mg} /$ day for 8 hours) with an interval of 1 week for the newly developed lesions. Although there was no clear improvement in the patient's neurological findings after the first cycle of rituximab, the tremor and weakness of the extremities and bulbar muscles gradually improved after the second cycle. Twenty days after the last rituximab treatment, she was discharged with improved neurological and psychiatric symptoms (Fig. 3). At 3-month follow-up, brain MRI showed a complete reversal of the occipital and basal ganglia signals (Fig. 4), and she resumed normal daily activity.

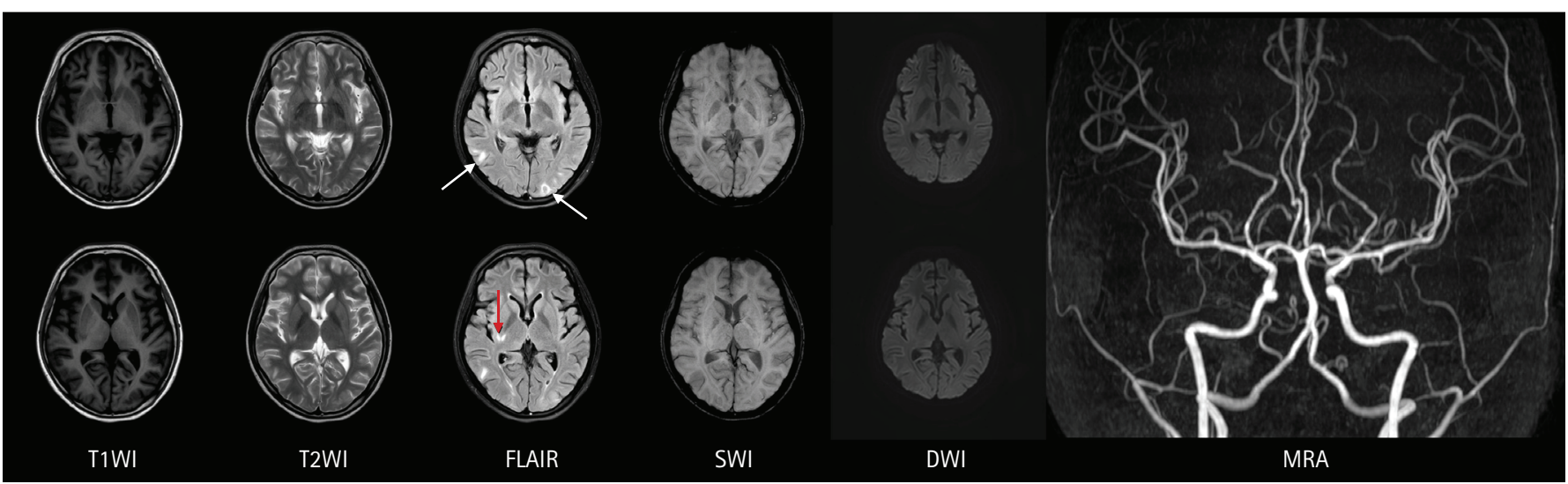

Fig. 2. Brain magnetic resonance imaging after intravenous methylprednisolone and immunoglobulin treatment. Fluid-attenuated inversion recovery (FLAIR) image revealed possible posterior reversible encephalopathy syndrome (white arrows) and possible lupus cerebritis in the right basal ganglia region (red arrow). Diffusion weighted imaging (DWI), susceptibility-weighted imaging (SWI), and timeof-flight magnetic resonance angiography (MRA) showed no evidence of ischemic and hemorrhagic vascular lesions.

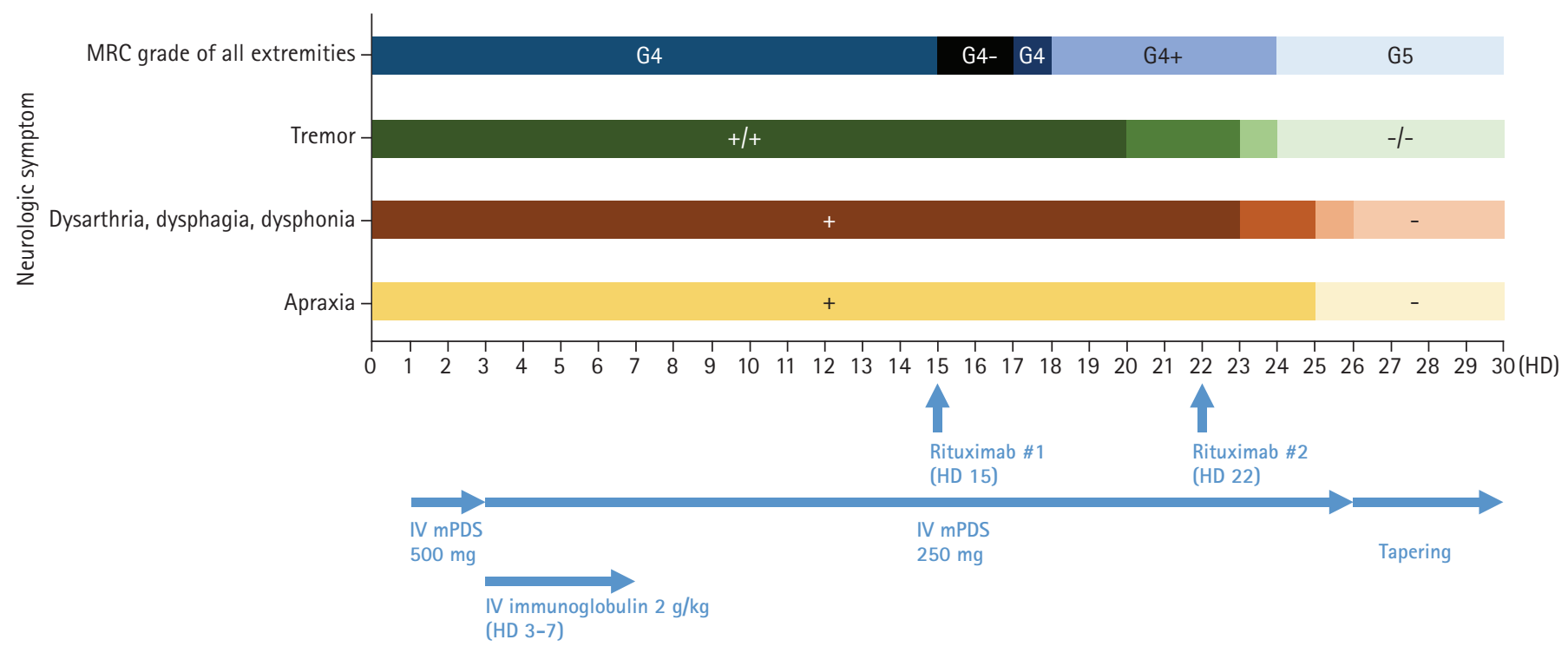

Fig. 3. Clinical course of the patient. MRC, Medical Research Council; HD, hospital day; IV, intravenous; mPDS, methylprednisolone. 

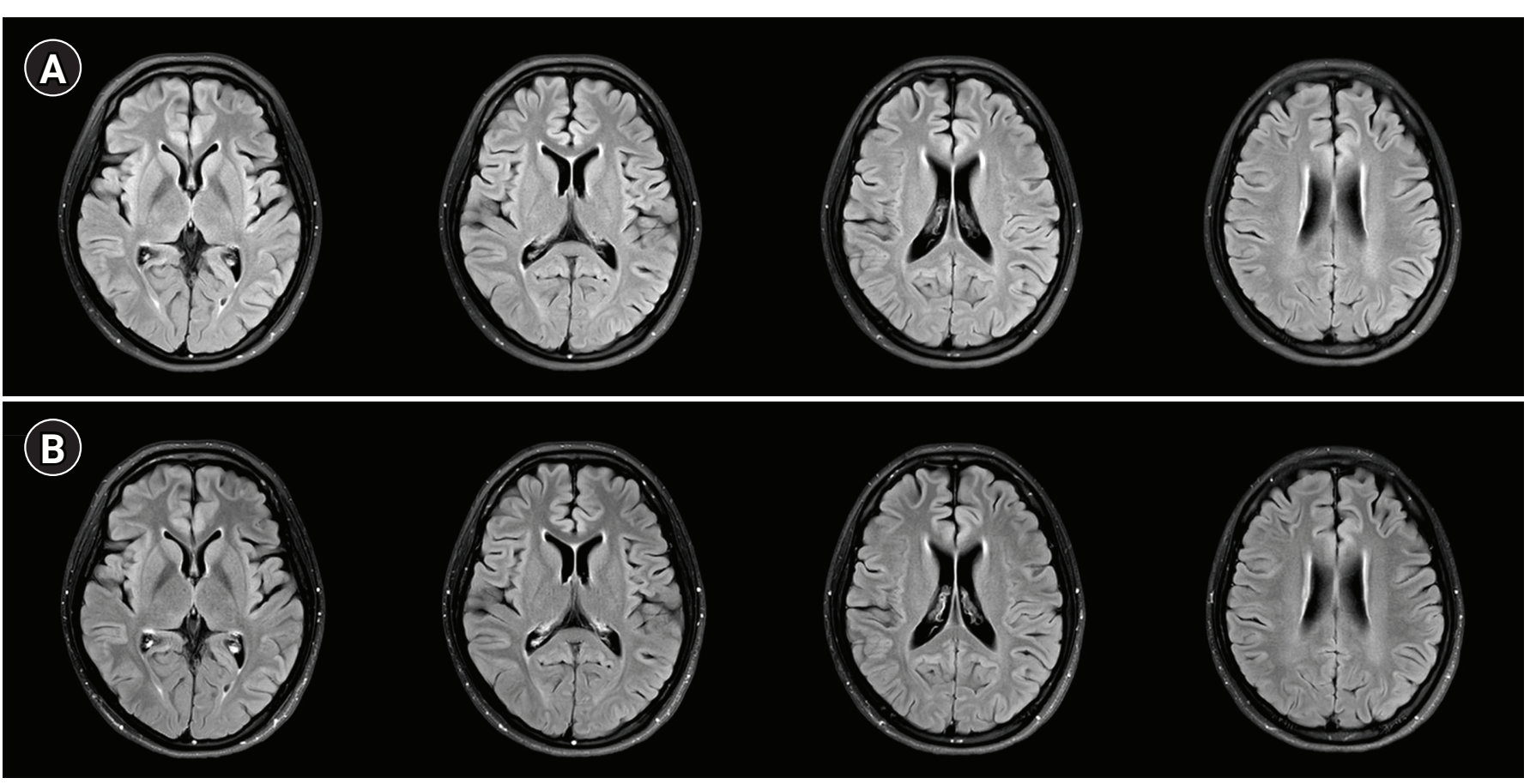

Fig. 4. Brain magnetic resonance imaging performed 3 months after rituximab treatment. Fluid-attenuated inversion recovery (FLAIR) image (A) and FLAIR-enhanced image (B) showed no abnormalities.

\section{DISCUSSION}

Here, we present the case of a young woman with lupus cerebritis and PRES who had been previously diagnosed with SLE. Unlike intravenous methylprednisolone and immunoglobulin, two cycles of intravenous rituximab successfully reversed her neurologic and psychiatric symptoms. Furthermore, her radiologic abnormalities along with neuropsychiatric symptoms were completely reversed 3 months after symptom onset.

Neuropsychiatric involvement in patients with SLE has been directly linked to SLE (primary NPSLE) or to other factors such as medicines, systemic diseases, and concomitant psychiatric disorders (secondary NPSLE) [6]. Although the pathogenesis of primary NPSLE is not fully understood, immune or inflammation-mediated blood brain barrier disruption with active generalized disease (inflammatory NPSLE) and thrombotic cerebrovascular disease with antiphospholipid antibody (thrombotic NPSLE) have been suggested to cause primary NPSLE $[7,8]$. Nevertheless, it is important to manage neuropsychiatric manifestations in SLE patients because they are associated with increased risk of death and worse functional outcome $[9,10]$. However, the diagnosis of NPSLE remains challenging even for experienced physicians because there is no specific biomarker for NPSLE. Therefore, researchers have attempted to developed various models or algorithms involving disease activity, imaging techniques, and CSF examinations to assist clinicians in diagnosing NPSLE [11]. Our patient was simultaneously diagnosed with lupus cerebritis (primary NPSLE) and PRES (secondary NPSLE) based on clinical findings, brain MRI, and CSF examination. Although rituximab is considered as second-line therapy for inflammatory NPSLE that shows no clinical response to methylprednisolone $[4,12]$, it was more common and easier to use intravenous immunoglobulin, which is consider as third-line therapy for inflammatory NPSLE, in our hospital. However, in this case, intravenous immunoglobulin was not effective, and it might have induced PRES. Nevertheless, we anticipated that immunotherapy against lupus cerebritis and symptomatic treatment with blood pressure lowering for PRES would reverse the patient's neuropsychiatric symptoms, and this proved to be the case.

The treatment of NPSLE is challenging, and no specific guidelines for the treatment of NPSLE have been established. This could be due to the obscure and complex pathophysiology of NPSLE as well as the fact that well-designed clinical studies are limited by small sample sizes [13]. Nevertheless, it is most important to diagnostically decide whether the neuropsychiatric symptoms are caused by primary NPSLE and the dominant category of NPSLE (inflammatory vs. thrombotic). An inflammatory cause is considered in case of young patients, NPSLE occurrence close to the time of SLE diagnosis, increased lupus activity or 
flare, antiphospholipid antibody-negative patients, worsening neuropsychiatric symptoms, NPSLE relapses, and abnormal results of CSF examination and brain MRI , and immunosuppressive treatment is recommended in such cases [13]. In inflammatory NPSLE, the first-line treatment of choice is high dose intravenous methylprednisolone. A randomized clinical trial demonstrated that intravenous cyclophosphamide $\left(0.75 \mathrm{~g} / \mathrm{m}^{2}\right)$ combined with methylprednisolone was more effective than intravenous methylprednisolone alone [14]. However, because cyclophosphamide is difficult to use and has many side effects, physicians generally hesitate to use it. Despite the lack of evidence from randomized clinical trials, B-cell depleting agents (rituximab; an anti-CD20 monoclonal antibody) are widely used to successfully treat refractory NPSLE in patients who do not respond to first-line therapy [15]. In this case, the patient's clinical symptoms also gradually improved to complete remission after two cycles of rituximab separated by an interval of 1 week (Fig. 4). Her brain MRI findings also improved 3 months after rituximab therapy.

Accurate diagnosis and treatment of NPSLE are crucial for managing every neuropsychiatric event in patients with SLE. Rituximab may be an effective treatment for refractory inflammatory NPSLE.

\section{ARTICLE INFORMATION}

\section{Ethics statement}

This study was approved by the Institutional Review Board of Hanyang University Hospital (IRB No. HYUH 2021-10-015). Informed consent from a patient was waived by the IRB.

\section{Conflict of interest}

No potential conflict of interest relevant to this article.

\section{ORCID}

Saemi Choi

https://orcid.org/0000-0002-7534-1243

Young Seo Kim

https://orcid.org/0000-0002-7050-3426

\section{Author contributions}

Conceptualization: YSK. Data curation: SC. Formal analysis: SC. Methodology: YSK. Project administration: YSK. Visualization: SC. Writing-original draft: SC. Writing-review \& editing: YSK.

\section{REFERENCES}

1. Govoni M, Bortoluzzi A, Padovan M, Silvagni E, Borrelli M, Donelli $\mathrm{F}$, et al. The diagnosis and clinical management of the neuropsychiatric manifestations of lupus. J Autoimmun 2016; 74:41-72.

2. The American College of Rheumatology nomenclature and case definitions for neuropsychiatric lupus syndromes. Arthritis Rheum 1999;42:599-608.

3. Schwartz N, Stock AD, Putterman C. Neuropsychiatric lupus: new mechanistic insights and future treatment directions. Nat Rev Rheumatol 2019;15:137-52.

4. Carrión-Barberà I, Salman-Monte TC, Vílchez-Oya F, Monfort J. Neuropsychiatric involvement in systemic lupus erythematosus: a review. Autoimmun Rev 2021;20:102780.

5. Muhammad O, Jindal H, Sharath M, Khan AM, Choi S. Systemic lupus erythematosus with multi-organ involvement in a young female: lymphadenopathy, lupus cerebritis, lupus nephritis, and cardiac manifestations. Cureus 2021;13:e15517.

6. Bertsias GK, Tektonidou M, Amoura Z, Aringer M, Bajema I, Berden JH, et al. Joint European League Against Rheumatism and European Renal Association-European Dialysis and Transplant Association (EULAR/ERA-EDTA) recommendations for the management of adult and paediatric lupus nephritis. Ann Rheum Dis 2012;71:1771-82.

7. Hanly JG. Diagnosis and management of neuropsychiatric SLE. Nat Rev Rheumatol 2014;10:338-47.

8. Bertsias GK, Boumpas DT. Pathogenesis, diagnosis and management of neuropsychiatric SLE manifestations. Nat Rev Rheumatol 2010;6:358-67.

9. Ahn GY, Kim D, Won S, Song ST, Jeong HJ, Sohn IW, et al. Prevalence, risk factors, and impact on mortality of neuropsychiatric lupus: a prospective, single-center study. Lupus 2018; 27:1338-47.

10. Kwon HS, Kim YS, Lee JM, Koh SH, Kim HY, Kim C, et al. Causes, risk factors, and clinical outcomes of stroke in Korean young adults: systemic lupus erythematosus is associated with unfavorable outcomes. J Clin Neurol 2020;16:605-11.

11. Bortoluzzi A, Scirè CA, Bombardieri S, Caniatti L, Conti F, De Vita $S$, et al. Development and validation of a new algorithm for attribution of neuropsychiatric events in systemic lupus erythematosus. Rheumatology (Oxford) 2015;54:891-8.

12. Magro-Checa C, Zirkzee EJ, Huizinga TW, Steup-Beekman GM. Management of neuropsychiatric systemic lupus erythematosus: current approaches and future perspectives. Drugs 2016;76:459-83.

13. Nikolopoulos D, Fanouriakis A, Bertsias G. Treatment of neuropsychiatric systemic lupus erythematosus: clinical challenges and future perspectives. Expert Rev Clin Immunol 2021; 17:317-30.

14. Barile-Fabris L, Ariza-Andraca R, Olguín-Ortega L, Jara LJ, Fra- 
ga-Mouret A, Miranda-Limón JM, et al. Controlled clinical trial of IV cyclophosphamide versus IV methylprednisolone in severe neurological manifestations in systemic lupus erythematosus. Ann Rheum Dis 2005;64:620-5.
15. Tokunaga M, Saito K, Kawabata D, Imura Y, Fujii T, Nakayamada S, et al. Efficacy of rituximab (anti-CD20) for refractory systemic lupus erythematosus involving the central nervous system. Ann Rheum Dis 2007;66:470-5. 